\title{
Significados e vivências de pacientes que convivem com psoríase
}

\author{
Meanings and experiences Of patients living with psoriasis \\ Significados y experiencias de los pacientes que viven con psoriasis
}

Junia Patrícia Pereira Durães Alves

ORCID: https://orcid.org/0000-0001-7912-2825

Faculdades Unidas do Norte de Minas Gerais, Brasil E-mail:juniappenf@gmail.com

Keila Santos Silva

ORCID: https://orcid.org/0000-0003-0493-0654 Faculdades Unidas do Norte de Minas Gerais, Brasil E-mail:keilasantosilva10@gmail.com

Leila das Graças Siqueira

ORCID: https://orcid.org/0000-0003-2907-5690 Faculdades Unidas do Norte de Minas Gerais, Brasil

E-mail: leiladasgsiqueira@gmail.com

Kênia Emanuelle Aguiar Fagundes

ORCID: https://orcid.org/0000-0003-1431-4257

Faculdades Unidas do Norte de Minas Gerais, Brasil

E-mail: keniaemanuelle.aguiarenf@gmail.com

Davidson Gonçalves Soares

ORCID: https://orcid.org/0000-0002-3014-0923

Faculdades Unidas do Norte de Minas Gerais, Brasil

E-mail: davidson.gsoares@gmail.com

Pedro Henrique Souza Reis

ORCID: https://orcid.org/0000-0003-4855-606X Faculdades Unidas do Norte de Minas Gerais, Brasil

E-mail: pedrohsouzareis.med@gmail.com

Gregório Ribeiro de Andrade Neto

ORCID: https://orcid.org/0000-0002-1417-5237

Faculdade de Saúde Ibituruna, Brasil

E-mail: gregorio.ribeiroenf@gmail.com

Kísia Kelly Ferreira Rosa

ORCID: https://orcid.org/0000-0001-7279-942X

Faculdades Unidas do Norte de Minas Gerais, Brasil

E-mail:kisiakellyfenfermagem@gmail.com

Fernanda Fagundes Fernandes

ORCID: https://orcid.org/0000-0002-3451-6537

Faculdades Unidas do Norte de Minas Gerais, Brasil

E-mail: fernandafagundes.f10@gmail.com

Robson de Souza França Ramos

ORCID: https://orcid.org/0000-0003-4457-5941

Faculdades Unidas do Norte de Minas Gerais, Brasil

E-mail: robsondesouza.enf@gmail.com

Júlio César Figueiredo Junior

ORCID: https://orcid.org/0000-0003-3406-1234

Faculdade de Saúde Ibituruna, Brasil

E-mail: juliocfjunior@gmail.com

Ana Amélia Alkmim Santos Torres

ORCID: https://orcid.org/0000-0001-7903-8965

Faculdades Unidas do Norte de Minas Gerais, Brasil

E-mail: anaameliaalkminmed@gmail.com

Ariane Teixeira Andrade

ORCID: https://orcid.org/0000-0001-7981-5074

Universidade Federal dos Vales do Jequitinhonha e Mucuri, Brasil

E-mail: arianeteixeira.andrade.moc@hotmail.com

Sylmara Corrêa Monteiro

ORCID: https://orcid.org/0000-0003-4546-336X Instituto Federal de Educação, Ciência e Tecnologia do Norte de Minas Gerais, Brasil

E-mail: scmenfermagem9@gmail.com

Natália Gonçalves Ribeiro

ORCID: https://orcid.org/0000-0001-7596-3846

Faculdades Unidas do Norte de Minas Gerais, Brasil

E-mail: natalia.gribeiro@gmail.com 


\begin{abstract}
Resumo
Objetivo: Objetivou-se compreender o significado das vivências de pacientes que convivem com psoríase. Realizou-se uma pesquisa de natureza qualitativa utilizando um roteiro não estruturado. Participaram da pesquisa pacientes com diagnóstico de psoríase cadastrados e acompanhados no ambulatório de dermatologia de uma Policlínica do Sistema Único de Saúde em um município localizado no norte de Minas Gerais. As respostas obtidas foram agrupadas de forma a atender os objetivos e categorizadas para viabilizar a discussão utilizando a técnica do discurso do sujeito coletivo. Os resultados revelaram que além das características clínicas da psoríase, as condições psicológicas e satisfação quanto à aparência física são fatores que interferem no impacto da doença sobre a qualidade de vida. Tais dados apontam para as peculiaridades clínicas da doença e do funcionamento psicológico, influenciando a adaptação psicossocial a pacientes com psoríase. Espera-se que esta pesquisa possa contribuir para os aspectos e mudanças na vida de pessoas com a doença, conscientizando a sociedade e a família da importância de acolher, cuidar e respeitar o indivíduo.
\end{abstract}

Palavras-chave: Psoríase; Dermatopatias; Avaliação em saúde; Percepção.

\begin{abstract}
Objective: The objective was to understand the meaning of the experiences of patients living with psoriasis. A qualitative research was carried out using an unstructured script. Patients diagnosed with psoriasis registered and followed at the dermatology outpatient clinic of a Polyclinic of the Unified Health System in a municipality located in the north of Minas Gerais participated in the research. The answers obtained were grouped in order to meet the objectives and categorized to enable the discussion using the collective subject's discourse technique. The results revealed that in addition to the clinical characteristics of psoriasis, psychological conditions and satisfaction with physical appearance are factors that interfere in the impact of the disease on quality of life. These data point to the clinical peculiarities of the disease and psychological functioning, influencing psychosocial adaptation to patients with psoriasis. It is expected that this research can contribute to the aspects and changes in the lives of people with the disease, making society and the family aware of the importance of welcoming, caring for and respecting the individual.
\end{abstract}

Keywords: Psoriasis; Dermatopathies; Health assessment; Perception.

\title{
Resumen
}

Objetivo: El objetivo fue comprender el significado de las experiencias de los pacientes que viven con psoriasis. Se realizó una investigación cualitativa utilizando un guión no estructurado. Participaron de la investigación pacientes diagnosticados de psoriasis registrados y seguidos en el ambulatorio de dermatología de un Policlínico del Sistema Único de Salud de un municipio ubicado en el norte de Minas Gerais. Las respuestas obtenidas fueron agrupadas para cumplir con los objetivos y categorizadas para posibilitar la discusión utilizando la técnica del discurso del sujeto colectivo. Los resultados revelaron que además de las características clínicas de la psoriasis, las condiciones psicológicas y la satisfacción con la apariencia física son factores que interfieren en el impacto de la enfermedad en la calidad de vida. Estos datos apuntan a las peculiaridades clínicas de la enfermedad y el funcionamiento psicológico, influyendo en la adaptación psicosocial a los pacientes con psoriasis. Se espera que esta investigación pueda contribuir a los aspectos y cambios en la vida de las personas con la enfermedad, haciendo que la sociedad y la familia sean conscientes de la importancia de acoger, cuidar y respetar al individuo.

Palabras clave: Psoriasis; Dermatopatías; Evaluación de la salud; Percepción.

\section{Introdução}

Etiologicamente desconhecida, a psoríase, trata-se de uma doença inflamatória crônica em que a pele do indivíduo é afetada, sendo ela congênita e não contagiosa. Assim na psoríase a epiderme produz células em uma velocidade acelerada e o desenvolvimento anormal dessas células faz com que se desprendam ocasionando lesões cutâneas. Estes transtornos celulares acelerados, por sua vez, não deixam que o processo normal do desenvolvimento dessas células ocorra, impedindo a função da pele que é a proteção celular, através de suas camadas (Marques, \& Rodrigues, 2011; Holbach, Brum, \& Lendengues 2013).

A psoríase ocorre frequentemente no couro cabeludo e superfícies de extensão de joelhos e região dos cotovelos, embora, qualquer região da pele pode ser afetada pelas lesões. As unhas, são, comumente atingidas, e traumatismos de qualquer etiologia, que afetam a pele do paciente, podem acarretar o surgimento de novas lesões na área afetada. O espalhamento por toda a pele é conhecido como forma entrodérmica, são conhecidas também na literatura as formas clínicas vulgar, artropática e eritrodérmica, essas duas últimas são as formas de controle mais complexas (Proença \& Maia, 1995). Essas modalidades clínicas resultam em muitas dificuldades em situações sociais, nas quais ocorre exposição de maneira parcial ou total do corpo do indivíduo (Ginsburg \& Link, 1988; Picardi \& Abeni, 2001). 
As causas e agravantes que podem desencadear a doença estão relacionados a período de estresse, ansiedade e/ou trauma vivido pela pessoa com a doença, enfim, tudo que engloba o sistema psicossocial e emocional do indivíduo pode desencadear e consequentemente agravar o quadro clínico, pode ocorrer devido a constituição genética, uma vez que a psoríase é hereditária, além disso, o sistema imune é estimulado ocasionando baixa de leucócitos deixando o indivíduo ainda mais exposto a outras doenças (Marques, \& Rodrigues, 2011; Holbach, Brum, \& Lendengues 2013).

As características da psoríase não têm sido bem esclarecidas, afetando cerca de 3\% da população mundial com partes iguais entre o sexo masculino e feminino, crianças também podem apresentar um quadro de psoríase durante a infância permanecendo periodicamente ao logo de sua vida adulta, tendo manifestações entre a segunda e terceira década de vida (Marques, \& Rodrigues, 2011; Holbach, Brum, \& Lendengues 2013).

Existem diferentes classificações para a psoríase e estas classificações dependerá da forma na qual se manifesta esta lesão. Os locais que mais são atingidos pela doença consequentemente são: o couro cabeludo, as superfícies extensoras dos cotovelos e joelhos, partes inferiores das costas e genitálias, podendo ainda se manifestar em unhas, e regiões palmares e plantares de mãos e pés (sendo essas as que mais descamam e apresentam fissuras, rachaduras e sangramentos constantes) (Holbach, Brum, \& Lendengues 2013).

A história da doença varia individualmente, no entanto, de forma geral, assume um perfil crônico, muitos pacientes podem cursar com diversas modalidades de recidiva e agravamento do quadro clínico. Para que a doença se manifeste, é preciso a presença de alguns fatores, cuja relevância é influenciada de forma individual. Entre esses, pode ser indicado irritação da pele resultado da pressão, traumas e queimaduras por exposição à luz solar, uso crônico de bebidas alcoólicas, estresse físico, alguns fármacos, infecções de aspecto crônico ou obesidade (Bork \& Brauninger, 1998).

Salienta-se a relevância de diagnosticar a psoríase de forma inequívoca, pois, frequentemente, o quadro pode ser avaliado de forma incorreta, uma vez que outras doenças podem acarretar placas e descamação semelhantes. À proporção que a psoríase se agrava, o padrão descamativo singular é comumente de fácil reconhecimento pelo profissional médico e, por esse motivo, os exames diagnósticos não são frequentemente exigidos. Contudo, para a confirmação do diagnóstico, o médico pode solicitar a biópsia de pele (Berkow, Beers, Bogin, \& Fletcher, 2003). Outra variável que merece destaque é a habilidade do paciente em lidar com a doença, ou seja, sua capacidade de adaptação para o enfrentamento e desenvolvimento de ferramentas que permitam controlar os sinais e sintomas da doença (Silva, 2003).

Nessa discussão, a integração entre a assistência dermatológica e psicológica só causa efeitos positivos para os pacientes com psoríase, quando se busca também o tratamento farmacológico, no entanto, nem sempre isso ocorre, pois, na maioria dos casos, os pacientes abandonam o tratamento, pois esperam uma solução imediata para as barreiras interpessoais, o que não ocorre frequentemente, pois o controle do quadro clínico sofre influência dos aspectos psicológicos (Guedes \& Ferreira, 2020).

Apesar da doença ter alguns sinais e sintomas que são visíveis e ser uma inflamação de caráter exclusivamente epidérmico e não contagioso, o indivíduo acometido, queixa-se de receios quando é necessário expor seu corpo, muitos deles, desistem do tratamento dermatológico e frequentemente do tratamento farmacológico, tendo sua vida social, profissional e afetiva prejudicadas pela manutenção das barreiras nessas áreas de suas vidas (Ginsburg \& Link, 1988).

As demonstrações de preconceito à imagem física dos pacientes com psoríase também podem ser mais veladas ou menos evidentes, como por exemplo, evitar o toque e a proximidade física com pessoas que possuam a doença, tais como, sentarse junto, o ato do abraço, pegar na mão, compartilhar sabonetes e toalhas, divisão de objetos, ou o indivíduo com psoríase ser alvo de piadas por colegas de trabalho, gerando constrangimento ou por fim o afastamento total (Mahoney, Cairns, Beverley \& Farmer, 2003). 
Em relação ao bem-estar psíquico de pacientes com psoríase, os indivíduos que sofrem com a psoríase geralmente ficam totalmente vulneráveis e abalados, uma vez que estes indivíduos tendem a ter sua autoestima baixa por se isolarem em meio a preconceitos sofridos diante da falta de informação da sociedade em relação à doença, que por sua vez é um problema que afeta as áreas mais expostas da superfície dérmica, se tornando visivelmente percebidas (Marques, \& Rodrigues, 2011). Nesse sentido, o presente estudo teve o objetivo de compreender o significado das vivências de pacientes que convivem com psoríase.

\section{Metodologia}

Este estudo caracteriza-se como pesquisa de cunho qualitativo e de abordagem descritiva, o que segundo Minayo (2013) lida com o universo de significados na perspectiva do outro, sendo baseada na subjetividade e na percepção de fenômenos do indivíduo.

A pesquisa foi realizada inicialmente junto ao ambulatório de dermatologia de uma policlínica pertencente a rede assistencial do Sistema Único de Saúde e localizada em um município do Norte de Minas Gerais. Inicialmente, os pesquisadores realizaram a busca acerca do perfil dos pacientes acompanhados na policlínica, bem como a relação numérica dos pacientes, assim, obteve-se o registro de seis pacientes.

E, logo, após identificação dos participantes entrou-se em contato com os mesmos por meio de visita domiciliar para agendar data e horário para a coleta dos dados. A coleta de dados ocorreu no domicílio de 3 dos 6 entrevistados, pois, 3 deles recusaram-se a participar da pesquisa, dessa forma, o processo de amostragem foi por conveniência.

Foi utilizada a técnica de entrevista com uso de um roteiro semiestruturado contendo questões norteadoras sobre o tema, os participantes discorreram livremente sobre o tema proposto. Foi assegurado a privacidade e o conforto do paciente, de forma que foi indicado no domicílio o espaço mais confortável para responder as perguntas.

Foi garantido anonimato, bem como, foi solicitada a permissão para gravar as falas e a assinatura do termo de consentimento livre e esclarecido (TCLE). Para preservar o anonimato dos participantes, eles foram codificados pela letra E de entrevistado seguido de número arábico obedecendo à ordem de coleta dos dados.

A análise dos dados baseou-se na Técnica do discurso do sujeito coletivo que é uma técnica de construção do pensamento coletivo que visa revelar como as pessoas pensam, atribuem sentidos e manifestam posicionamentos sobre determinado assunto. Trata-se de um compartilhamento de idéias dentro de um grupo social, entende-se por discurso todo posicionamento argumentando (Lefèvre, 2005).

$\mathrm{Na}$ construção do discurso do sujeito coletivo são utilizados trechos do discurso, que foram descrições literais dos depoimentos, reveladores da essência do conteúdo das representações e que são denominados de expressões-chave. A partir dos recortes de fala significativos são identificadas as ideias centrais, ou seja, as palavras ou expressões lingüísticas que revelam, de maneira precisa e sintética, o sentido presente nos depoimentos. Organizam-se, em mapas denominados de Instrumentos de Análise do Discurso (IAD), as expressões-chave e as ideias centrais. As expressões-chave exemplificadoras de idéias centrais semelhantes são organizadas de forma lógica e coerente, compondo um discurso único redigido na primeira pessoa do singular: o discurso do sujeito coletivo (Lefèvre, 2005).

Quanto aos aspectos éticos da pesquisa, esta foi realizada mediante autorização do Comitê de Ética em Pesquisa da Associação Educativa do Brasil - SOEBRAS conforme parecer consubstanciado $\mathrm{N}^{\circ} 2.018 .753$, em atendimento ao disposto na Resolução 466 do Conselho Nacional de Saúde (Brasil, 2012). 


\section{Resultados e Discussão}

Pelos depoimentos, foi possível compreender o significado e as vivências de pacientes que convivem com psoríase e os impactos da doença na sua vida familiar e pessoal. A psoríase é uma doença que apresenta vários fatores relacionados à dificuldade de controle da doença causando limitações físicas e psíquicas resultando em barreiras psicossociais, comprometendo a participação social dessas pessoas na família, trabalho e lazer, levando a um forte estigma social na vida daqueles que sofrem desta doença (Marques, \& Rodrigues, 2011; Holbach, Brum, \& Lendengues 2013).

São comuns relatos dos usuários com psoríase relacionado a problemas com a imagem corporal, sentimentos de vergonha de sair em lugares públicos devido às lesões provocar constrangimentos, sendo confundida com outras doenças, acarretando o isolamento, a diminuição da autoestima e privação de participação social (Marques, \& Rodrigues, 2011; Holbach, Brum, \& Lendengues 2013).

Do ponto de vista clínico, a psoríase vulgar é a mais frequente. Essa modalidade é caracterizada por pápulas e placas de coloração rósea ou avermelhadas que podem assumir vários tamanhos, sendo bem delimitadas, de aspecto seco e frequentemente cobertas com camadas de finas escamas de tom prateado. O couro cabeludo, a região sacra e as superfícies extensoras das partes distais são comumente afetados, embora em alguns indivíduos as áreas flexurais e intertriginosas sejam, sobretudo, afetadas (Elder, Elenitsas, Ioffreda, Miller, Miller, \& Johnson, 2001).

A psoríase é uma doença que não tem cura, pois os pacientes podem até melhorar o quadro clínico, mas ela pode vir a rescindir por várias vezes ao longo da vida. Ela também pode provocar incapacidades, assim como a diabetes e a asma, mas sua prevalência depende muito de cada indivíduo, porque está diretamente ligada ao estilo de vida pessoal e ao modo de enfrentamento da doença (Holbach, Brum, \& Lendengues 2013).

Em relação ao tratamento, quando o paciente apresenta apenas algumas placas pequenas, a psoríase responde de forma rápida a intervenção, como por exemplo, o uso de pomadas e cremes emolientes que auxiliam na umidificação da pele. Pomadas com corticóides, colecalciferol, ácido salicílico podem também ser indicados. A luz ultravioleta e a exposição ao sol também podem ser uma forma de tratamento que pode auxiliar na eliminação dos sintomas da psoríase. Entretanto, a terapêutica com raios ultravioletas pode elevar o risco de câncer de pele em razão da luz ultravioleta e, por esse motivo, o tratamento deve ser supervisionado de forma rigorosa por um profissional médico (Richards, Fortune, Griffiths \& Main 2001).

A psoríase pode ainda causar incapacidades, ocasionando diabetes, asma, a artrite psoriática, também propicia problemas cardiovasculares, sendo que a prevalência dos mesmos dependerá de cada indivíduo e de seu estado físico e emocional, uma vez que tem relação direta com o estado físico e psicológico do paciente (Marques, 2011, Lipp, 2003, Steiner \& Perfeito, 2003).

A falta de conhecimento sobre a doença gera nas pessoas sentimentos de preconceito em relação aos pacientes de psoríase, por causa de suas lesões, por isso é importante conscientizar as pessoas que a psoríase não é infecciosa, não é ausência de higiene pessoal ou câncer de pele, assim essa doença deixa marcas sentimentais na vida das pessoas, pois muitas das pessoas que foram diagnosticadas não têm informação completa da doença. Fato este que pode ser observado nas falas dos entrevistados abaixo:

"São umas manchinhas que às vezes aparecem e logo somem, às vezes preciso fazer uso de algum medicamento" E1.

"O que é isso? E eu mesmo não sei explicar o que é, sei que me falaram que era psoríase, só sei que tenho esse incômodo" E2.

"Saiu uma coisa aqui na orelha, aqui do lado do braço, mas que negócio é esse que não sara?" E3. 
Os dados revelam que o conhecimento dos pacientes em reação à doença é muito escasso, muitos recebem o diagnóstico e na maioria das vezes nem sabe do que se trata, no entanto, todos sabem que se trata de uma doença que não tem cura, pois os pacientes podem até melhorar o quadro clínico, mas ele pode vir a rescindir por várias vezes ao longo da vida.

A falta de conhecimento sobre a doença gera nas pessoas sentimento de preconceito em relação aos pacientes de psoríase, por causa de suas lesões, por isso é importante conscientizar as pessoas que a psoríase não é infecciosa, não é ausência de higiene pessoal ou câncer de pele, pois, a aparência geral das lesões é caracterizada com o aparecimento de escamas prateadas grossas que sobressaem à pele lesionada, quando se desprendem dessa pele deixam expostas placas em forte rubor que levam a fissuras e rachaduras no percurso de toda lesão (Holbach, 2013, Marques, \& Rodrigues, 2011).

As falas abaixo permitem descrever que a maioria dos pacientes que sofrem com a psoríase apresenta sentimentos negativos, geralmente ficam totalmente abalados e vulneráveis e tendem a ter uma baixa autoestima:

"Preconceito eu sofro sim, pois as pessoas sempre ficam olhando e perguntando" E1.

"Eu tiro isso como uma estupidez sem tamanho, eu explico que isso é psoríase, que não pega, porque o olhar de repulsa é demais" E2.

"Uma vez eu fui pegar um ônibus e a cobradora veio pegar meu dinheiro pela pontinha dos dedos com medo de ser uma doença contagiosa" E3.

"Às vezes dá vontade de falar assim: você deve ser muito mais doente do que eu! Porque a ignorância é um absurdo" E3.

Os pacientes que sofrem de psoríase tendem a tendem a ter uma baixa autoestima (Marques, 2011). Grande parte dos indivíduos com psoríase já sofreram situações de discriminação por causa de terem psoríase, este fator é relevante no aspecto psicossocial destes indivíduos, pois os pacientes também relataram sentimentos negativos acerca da aparência física e de discriminação social. Indivíduos com psoríase apresentam estresse e dificuldades em seu local de trabalho, relacionamento interpessoal, emprego e no uso de transporte e áreas de lazer. Essas dificuldades diminuem a interação social, facilitando o isolamento e a depressão (Arruda, Nogueira, Oliveira, Pereira \& Silva, 2010).

O impacto da doença na vida do paciente é negativo, muitos se sentem discriminados pela sociedade e em seu local de trabalho, lazer e até mesmo no seu âmbito de convívio familiar. As mudanças de hábito de vida diária também proporcionam impactos negativos na vida do paciente como: maior número de banhos diários, modo de se vestir, dificuldade de se desenvolver no ambiente de trabalho levando ao afastamento do mesmo e dificuldade de novo emprego:

"Na escola no meu serviço, quando as crianças (alunos) perguntam eu sempre explico que é ligada ao nervoso e ao estresse. [...] Às vezes, eles (outras pessoas) perguntam quando saí na região da orelha, sempre perguntam o que é, perguntam se tem tratamento, se tem cura" E1.

"Eu uso mais calça, bermuda, eu só uso aqui dentro de casa, porque se eu saio, e o povo pergunta" [...] Eu nem trabalho mais, mudei minha vida, aí eu tive de aposentar, eu era tão trabalhador, mas falei com ela (esposa), que Deus quis assim, então assim eu vou vivendo [...] Eu não posso usar sapato" E2.

“Quantas vezes já pedi para morrer ao tomar banho? De dor. Já passei por situações em que meu filho teve que tirar minha camisa para mim porque eu não tive condições. Então eu não posso nem trabalhar. Eu até separei da minha exmulher por isso, então minha vida é sozinho" E3. 
Em estudo realizado por Marques e Rodrigues, (2011) Hospital das Clínicas da Faculdade de Medicina de Ribeirão Preto (FMRP- USP) no estado de São Paulo pode-se constatar que cerca de 60\% destes pacientes entrevistados apresentavam sentimentos negativos sentindo-se socialmente discriminados pela sociedade em seu local de trabalho, lazer e no ambiente familiar.

A psoríase é uma doença que altera o comportamento social dos doentes, devido ao seu aspecto e as lesões de pele muito visíveis. Ela está relacionada a um alto impacto psicossocial, decorrente do desenvolvimento da doença, a intensidade do prejuízo adaptativo pode variar de acordo com a percepção do paciente. Neste sentido, as condições psíquicas do paciente com psoríase podem desempenhar um papel importante na autopercepção quanto à condição física e na mediação da intensidade do impacto da doença no cotidiano (Flambo, 2006; Mingorrance, Loureiro, Okino, \& Foss, 2001; Root, \& Al'abadie, 1994).

A percepção frente à aparência física associa-se a um maior prejuízo nas atividades cotidianas e desadaptação social. Os pacientes insatisfeitos quanto à aparência física referem maior prejuízo nas atividades rotineiras e na qualidade geral de vida. Neste caso, a satisfação quanto a si próprio e imagem corporal parecem exercer maior influência na adaptação psicossocial do paciente do que a melhora concreta da lesão (Mingorrance, Loureiro, Okino, \& Foss, 2001).

\section{Conclusão}

Este estudo proporcionou compreender a percepção de pacientes com psoríase sobre a sua doença e os sentimentos vivenciados. O significado é intenso na vida dos pacientes, pois muitos sofrem preconceitos e são discriminados pela sociedade e familiares, necessitando em alguns casos fazer o uso de medicações antidepressivas. É essencial conscientização da população e também dos próprios pacientes em relação à doença, na maioria das vezes os mesmos não sabem o que está acontecendo com seu próprio corpo. Isso implicou na aceitação dos pacientes em participar do estudo, pois em razão de apresentarem sentimentos negativos, foi recusado responder as perguntas.

Além das características clínicas da psoríase, as condições psicológicas e satisfação quanto à aparência física são fatores que interferem no impacto da doença. Tais dados apontam para as peculiaridades clínicas da doença e do funcionamento psicológico, influenciando a adaptação psicossocial, e sugerindo a importância do suporte psicológico como elemento complementar no atendimento a pacientes com psoríase. Assim, espera-se que esta pesquisa possa contribuir para mudanças positivas na vida de pessoas com psoríase, conscientizando a sociedade e a família da importância de acolher, cuidar e respeitar a pessoa doente.

\section{Referências}

Arruda, C. S., Nogueira, E., Oliveira, M. S., Pereira, E. R., \& Silva, R. M. C. A. (2010). Avanços e desafios da enfermagem na produção científica sobre psoríase. Universidade Federal Fluminense. Escola de Enfermagem Aurora Afonso Costa. Niterói, RJ, 63(2), $264-273$.

Brasil. (2012). Resolução $n^{o} 466$, de 12 de Dezembro de 2012. <http://conselho.saude.gov.br/resolucoes/2012/Reso466.pdf>

Bork, K., \& Brauninger, W. (1998). Dermatologia clínica: diagnóstico e terapia. (2a ed.), Manole. 127-130.

Berkow R., Beers, M. H., Bogin, R. M., \& Fletcher, A. J. (2003). Manual Merck de informação médica: saúde para a família. Manole. $1033-1034$.

Flambó, P. A. D. G. (2006). Avaliação do bem-estar psicológico em sujeitos com psoríase. Instituto Superior de Psicologia Aplicada. Dissertação de mestrado em Psicologia da Saúde.

Daniele, R. G., \& Solange C. F. (2020). A trajetória de uma vida marcada pelo preconceito e exclusão social em decorrência do estigma da Psoríase: Relato de caso. Research, Society and Development, 9(10), 1-10.

Elder, D., Elenitsas, R., Ioffreda, M., Miller, J., Miller, O. F., \& Johnson, B. (2001). Histopatologia da pele de leve: manual e atlas. Monole. 93-100.

Ginsburg, I. H. \& Link, B. G. (1988). Feelings of stigmatization in patients with psoriasis. Journal of the American Academy of Dermatology. 53-63.

Holbach, F., Brum, J.F., \& Lendengues, L. (2013) Ficha terminológica multilingüe Projeto: Dermatologia para Tradutores. 
Research, Society and Development, v. 10, n. 10, e572101019165, 2021

(CC BY 4.0) | ISSN 2525-3409 | DOI: http://dx.doi.org/10.33448/rsd-v10i10.19165

Lipp, M. E. N. (2003). O modelo quadrifásico do stress. In M. E. N. Lipp (Org), Mecanismos neuropsicofisiológicos do stress: teoria e aplicação clínica. São Paulo: Casa do Psicólogo. 17-22.

Marques, P. P., \& Rodrigues, C. D. S. (2011) Qualidade de vida de pacientes com psoríase: avaliação do índice de incapacidade. São José do Rio preto- SP, Arq. Ciênc. Saúde, 18(2), 73-76.

Mahoney, J. L., Cairns, B. D., \& Farmer, T. W. (2003). Promoting interpersonal competence and educational success through extracurricular activity participation. Journal of Educational Psychology. 95(2), 409-418.

Mingorance, R. C., Loureiro, S. R., Okino, L., \& Foss, N. T. (2001). Patients with psoriasis: psychosocial adaptation and personality features. Medicina, 34(2), 315-324.

Minayo, M. C. S. (2013). O desafio do conhecimento: Pesquisa qualitativa em saúde. São Paulo: Hucitec, 385-416.

Picardi, A., \& Abeni, D. (2001). Can cognitive-behavioral therapy help patients with vitiligo? Archives in Dermatology, 137(6), 752-788.

Proença, N. G., \& Maia, M. (1995). Psoríase. In O. L. Ramos \& H.A. Rothschild. Atualização terapêutica: manual prático de diagnóstico e tratamento ed.17. Porto Alegre: Artes Médicas. 606-6070.

Richards, H. L., Fortune, D. G., Griffiths, C. E., \& Main, C. J. (2001). The contribuition of perceptions of stigmatization to disability in patients with psoriasis. Journal of Psychosomatic Research, 50(1), 11-15.

Root, S., Kent, G., \& Al'abadie, M. S. K. (1994). The relationship between disease severity, disability and psychological distress in patients undergoing PUVA tratment for psoriasis. Dermatology, 189(26), 234-237.

Silva, K. (2003). Stress em pacientes com psoríase Trabalho de conclusão de curso não-publicado, Universidade Camilo Castelo Branco.

Steiner, D., \& Perfeito, F. L. (2003). A relação entre stress e doenças dermatológicas. In M. E. N. Lipp, (Org.), Mecanismos neuropsicofisiológicos do stress: teoria e aplicação clínica. São Paulo: Casa do Psicólogo. 111-114. 